\title{
It's the postgraduate education ...!
}

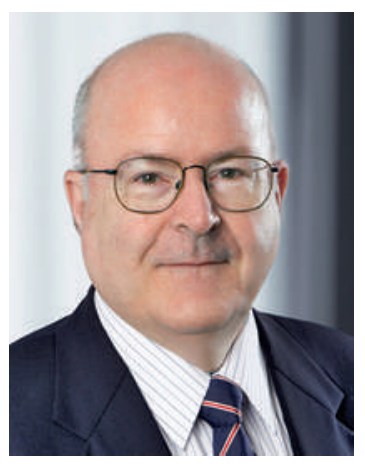

Im amerikanischen Wahlkampf erinnern sich Journalisten gerne geflügelter Worte, von denen man glaubt, dass sie wesentlich zum Wahlerfolg eines Kandidaten beigetragen haben. Einer dieser Slogans stammt von Bill Clinton: «It's the economy, stupid.»

So entscheidend die Wirtschaft für das Wohlergehen einer Gesellschaft ist, so entscheidend ist die Weiterbildung für das Gesundheitswesen. Deshalb ist es verlockend, das Zitat entsprechend umzuformulieren: «It's the postgraduate education (das Zusatzwort lassen wir weg) ...!»

Wir erleben ein Gesundheitswesen im Wandel, das den demographischen Entwicklungen, der Zunahme von chronischen Erkrankungen, den diagnostischen und therapeutischen Anforderungen der Polymorbidität und den limitierten personellen und finanziellen Mitteln gerecht werden muss. An Denkanstössen zur Bewältigung der heutigen und der sich abzeichnenden Probleme fehlt es nicht: «less is more», Stärkung der Hausarztmedizin oder «shared decision making» mit gut informierten Patienten.

\section{Für die Zukunft des Gesundheitswesens ist der Erhalt von genügend Weiterbildungsstellen eine indiskutable Notwendigkeit.}

Welche Konzepte sich auch als hilfreich erweisen mögen, etwas ist sicher: Die Arbeitslast und die Verantwortung der Ärztinnen und Ärzte werden auch mit interprofessionellen Arbeitsmodellen in Zukunft nicht geringer sein. Es ist zu hoffen, dass sie sich auf ihre wesentlichen Kompetenzen konzentrieren können, und dass zeitraubende Tätigkeiten wegfallen, welche andere Teammitglieder sinnvollerweise übernehmen können. Die ärztlichen Kompetenzen liegen in der Indikationsstellung für diagnostische und therapeutische Massnahmen, in der Koordination und Prioritätensetzung bei komplexen gesundheitlichen Problemen, in der Adaptation von Standards an den individuellen Patienten und in der Kommunikation mit den Patienten und im Team.

Dies alles muss man lernen!

Und deshalb: «It's the postgraduate education!»
Die Weiterbildung bis zum Facharzttitel legt die Basis für die qualitativ hochstehende Ausübung des Arztberufes. Für die Zukunft des Gesundheitswesens ist es entscheidend, dass eine hochkompetente Ärztegeneration bereit ist, die notwendigen Veränderungen zu gestalten und mitzutragen.

Für eine gute Weiterbildung braucht es neben begeisternden Weiterbildungsverantwortlichen Zeit und damit auch Geld. Der Arbeitsanfall und die vielfältigen, vor allem auch administrativen Belastungen nagen in manchen Weiterbildungsstätten an der Motivation der Kaderärztinnen und Kaderärzte, sich für das «Teaching» über das Mindestmass hinaus zu engagieren. Da und dort keimt denn - auch bei Spitaldirektoren - der Wunsch auf, Weiterbildungsstellen abzubauen und stattdessen Spitalfacharztstellen zu schaffen.

Auch wenn da und dort der Einsatz von Spitalfachärzten zweckmässig sein mag, muss hier klipp und klar festgehalten werden: Für die Zukunft des Gesundheitswesens ist der Erhalt einer genügenden Zahl von Weiterbildungsstellen eine indiskutable Notwendigkeit. Spitäler, die sich als «Weiterbildungs-Trittbrettfahrer» verstehen, sollten auf keine Spitalliste kommen. Ebenso klipp und klar muss hier unterstrichen werden, dass den Weiterbildungsverantwortlichen ausreichende finanzielle Mittel und genügend zeitliche Ressourcen zugesprochen werden müssen. Kantone und Spitäler stehen hier in der Pflicht - knappe Budgets hin, Marktaspekte her.

Auch das SIWF und die Fachgesellschaften sind gehalten, die Weiterbildung wo immer möglich zu unterstützen und sich Gedanken zu den anstehenden Massnahmen und Veränderungen zu machen. So werden dem Gesundheitswesen auch in Zukunft Ärztinnen und Ärzte zur Verfügung stehen, die ihren Aufgaben gewachsen sind.

Als Beispiel finden die Leserinnen und Leser in dieser Nummer der Ärztezeitung einen Bericht über drei Pilotworkshops, die im Herbst am Kantonsspital Luzern stattgefunden haben. Das Royal College of Physicians of London und das SIWF haben dieses Projekt gemeinsam entwickelt, das ganz dem Ziel «Teach the Teachers» verpflichtet war. Die interaktiven Workshops behandelten Themen wie «Assessment and feedback giving», «How to deal with an underperforming resident», «Teaching» und «Leadership». Angesichts des überwältigenden Erfolgs dieser Veranstaltung wird das SIWF im nächsten Jahr wieder eine Serie dieser Workshops anbieten, damit möglichst viele in der Weiterbildung Tätige ihre Erfahrung um neue Ideen erweitern können.

Dr. med. Werner Bauer,

Präsident des Schweizerischen Instituts für ärztliche Weiter- und Fortbildung SIWF 